\title{
Commentary on "Back to the Future": Narrative Treatment for Post-Traumatic, Acute Stress Disorder in the Case of Paramedic Mr. G
}

\section{"Back to the Future" Therapy: Its Present Relevance, Promise, and Implications}

\section{JACOB FARNSWORTH ${ }^{a}$ \& KENNETH W. SEWELL ${ }^{a, b}$}

\author{
${ }^{\mathrm{a}}$ University of North Texas, Department of Psychology, Denton, TX \\ ${ }^{\mathrm{b}}$ Correspondence concerning this article should be addressed to: Kenneth W. Sewell, Ph.D., Department of \\ Psychology, 1155 Union Circle \#311280, University of North Texas, Denton, TX 76203 \\ Email: kenneth.sewell@unt.edu
}

\begin{abstract}
Palgi and Ben-Ezra (2010) describe an intriguing approach to the treatment of acute stress responses in persons whose memory for a recent traumatic event has not yet consolidated-an approach they call "Back to the Future" therapy (BFT). The present article examines BFT on several dimensions: theoretical fidelity, applicability, and potential for empirical investigation. BFT shows considerable promise as a treatment for a subset of persons who experience a traumatic stressor. The greatest challenges now for users of BFT include both identifying persons most likely to benefit from BFT, and systematically evaluating the effectiveness of the approach.
\end{abstract}

Key words: posttraumatic stress; narrative psychotherapy; constructivist psychotherapy; Acute Stress Disorder (ASD); Post-Traumatic Stress Disorder (PTSD)

\section{OVERVIEW}

The preponderance of literature on treating traumatized individuals focuses on the clinical syndrome of Posttraumatic Stress Disorder (PTSD). Even therapeutic theorists and practitioners can easily forget that the "post-" to prefix to "traumatic" is an important specifier. In fact, applying many of the established approaches to peri-traumatic contexts (i.e., to a soldier still in the fox hole, or to an abused teenager still living in the home with the abuser) can endanger the physical and mental health of the traumatized person. Such interventions are explicitly designed for a POST-traumatic context. A less well developed literature exists on treating acute stress reactions that have not (yet?) developed into PTSD. That is where the Palgi and Ben-Ezra (2010) approach is firmly situated.

Palgi and Ben-Ezra (2010) propose that the symptoms included in Acute Stress Disorder (ASD) can be lessened or avoided by helping individuals recently confronted with traumatic 
events diffuse and integrate the traumatic event into their personal narrative. Their approach avoids explicit focus on the "traumatic nucleus" on the assumption that such a focus might inadvertently facilitate the consolidation of that nucleus and increase the possibility of PTSD. Instead, they focus on precursors and sequelae of the event (past and future). This "Back to the Future" therapy (BFT) attempts to re-script the traumatic event as something the individual was prepared for all along, thereby stripping it of its disruptive force. Furthermore, reducing the potency of the event's interpretation increases the chance that natural forgetting processes will allow the traumatic memory and its emotional sequelae to fade away, decreasing the likelihood of developing posttraumatic stress disorder . To this end, BFT advocates utilizing natural defense mechanisms to aid in the dissolution and forgetting of the core essence of the traumatic event before it can solidify.

To enable the re-narration of the traumatic event and allow natural defense mechanisms to minimize damage, Palgi and Ben-Ezra utilize White and Epston's (1990) concept of “unique outcomes" which the original authors defined as, "aspects of lived experience that fall outside the dominant story" (p. 15). As individuals create unitary and coherent personal narratives from the ongoing milieu of their lives, common themes are likely to be incorporated whereas unique or uncommon events are likely to be excluded as irrelevant or coincidental. Palgi and Ben-Ezra contend that certain of these unique outcomes (those that point to personal preparedness) present "anchoring points" with which to "challenge the client's basic assumptions" concerning their vulnerability to the trauma. It may need to be emphasized that "challenging" need not imply direct confrontation (a point not directly stated by Palgi and Ben-Ezra). Judging by the tone of their case example of Mr. G and the theoretical underpinnings of their approach, this “challenging” stance can be adopted by providing alternative interpretations and identifying unacknowledged information that, when considered, lead the client to profoundly different conclusions with regard to the traumatic event and its consequences for them.

Palgi and Ben-Ezra do an admirable job of reviewing the evidence regarding how memory is neuropsychologically constructed over a period of days and weeks, allowing for their proposed therapy to have its impact. However, their discussion regarding the centrality of narrative storying in the construction of healthy self-identify (the section entitled, "Narrative Psychotherapy as a Way of Changing the Traumatic Story") was remarkably brief. The reader who begins this presentation unconvinced of the importance of narrative will likely need to consult prior authors for a more thoroughgoing argument.

\section{DIRECTIVE OR COLLABORATIVE?}

BFT confronts therapists with two important realities: the autonomy of the client and the power of the therapist. We would propose that neglecting to acknowledge either of these realities can place the therapeutic relationship (and thus the client) at risk. One on hand, if the therapist fails to respect the client as the primary author for her/his own narrative, how can that same therapist argue that the client has developed resiliency (as opposed to simply becoming dependent upon the therapist to create meaning for her/him). On the other hand, neglecting to acknowledge the inherent power differential within a client-therapist relationship permits the therapist's power to influence the client without conscious and thoughtful prior consideration as to how such influence should be directed. 
Thus, it is important for the therapist employing BFT to ask, "Is this new story negotiated with or imposed upon the client?” To answer that it is negotiated implies something of a paradox. If it is negotiated, it must be questioned whether discussing the goals of the BFT will negate its efficacy by communicating to the client that the therapist's reinterpretations of their history are really just that-reinterpretations by the therapist that might or might not be "real" for the client. With such an explanation, the power of the alternative narrative presented by the therapist may be lessened and the client less willing to accept anchoring points as valid elements of the personal narrative. Conversely, if the therapist views her/himself as unilaterally imposing a new, resilient narrative on the client, it provokes theoretical (if not ethical) concerns regarding the client's role as the author of her/his own narrative and whether the therapist's influence is being over-applied (McAdams, 2006). Although Palgi and Ben-Ezra appear not to address this issue directly, their presentation of BFT seems content to allow the therapist to intervene as the primary, albeit temporary, author in the individual's story rather than as audience. The therapist appears to be given license by BFT to rewrite the narrative as to how the therapist would like the traumatic event to be presented and how the therapist would like to see the story end. Although this somewhat directive nature of BFT may provide some efficiency, the approach may face potential tradeoffs and difficulties as a result.

One potential pitfall that such a directive approach might encounter is negative reactions from at least some clients as the therapist attempts to take the reins, even subtly, of their narrative and rewrite resilience into it. Such an attempt might be construed by the client as disregarding or minimizing her/his personal experience of terror and horror. Given that perceived negative social interactions subsequent to traumatic events have been found to predict later posttraumatic symptoms (Borja, Callahan \& Long, 2006; Ullman \& Fillipis, 2001), this seems like an important issue for advocates of this approach to address in detail.

As a directive approach, the client-therapist power relationship in BFT may receive illumination from George Kelly’s fixed-role therapy (Kelly, 1955/1991). Much like BFT, Kelly’s fixed-role therapy includes the creation of an alternate script from which the client can experience the events of their life, and even adopt elements of the new role should they find them preferable. In fixed-role therapy, it is important to note that the therapist creates the client's alternative role in the context of group supervision and collaboration. As a result, the new role that is created is not the sole product of the therapist's vision of health, but instead a conceptual "average" of several perspectives on what might be useful for the client. In the case study presented, Palgi and Ben-Ezra mention the process of group discussion of the client's anchoring points, but do not provide details about the necessity for such collaboration. In addition to generating helpful feedback, incorporating consultation as a standard element of BFT might help to allay fears that the therapist is assuming too much power or being unduly directive in reforming the client's personal narrative.

Despite the foregoing critique, it remains possible that Palgi and Ben-Ezra intend a much more collaborative, negotiated re-narration process than our reading led us to infer. If such is the case, then these concerns might be ill-founded-and we invite further dialogue and clarification in future literature. 
"Back to the Future" Therapy: Its Present Relevance, Promise, and Implications

J. Farnsworth \& K.W. Sewell

Pragmatic Case Studies in Psychotherapy, http://pcsp.libraries.rutgers.edu

Volume 6, Module 1, Article 2, pp. 27-33, 02-27-10 [copyright by authors]

\section{APPROPRIATE CONTEXTS AND POPULATIONS FOR BFT}

Because each formal therapy approach is designed to address specified areas of concern, it becomes important to address for which contexts and populations a particular approach might or might not be useful. One of the core distinguishing features of ASD, dissociation, may also predispose clients to be more responsive to therapeutic suggestion (Eisen, 2001). Thus, a directive approach with BFT may be more effective for these suggestible individuals in reducing the encoding of traumatic memories or offering alternative personal narratives in which to integrate the traumatic event. Individuals more responsive to directive approaches, such as Palgi and Ben-Ezra’s (2010) case of Mr. G, whom they describe as “compliant”, may be ideally receptive to having the therapist reframe past life events for them as anchors. However, identifying this type of individual following a traumatic event might be problematic (see the section on "Investigating BFT" below)._On the other hand, clients who have been diagnosed with full-blown PTSD, but whose traumatic experiences have never fully solidified (despite considerable time since the traumatic event), may also benefit from an approach like BFT that diffuses - rather than elaborates — the traumatic nucleus.

Conversely, it is important to consider in which contexts and for which populations BFT may not be a viable treatment option. For instance, individuals with personal narratives emphasizing autonomy might find the directive attempt of BFT to re-narrate their past as invasive and invalidating. With such clients, it may be helpful to discuss anchoring points in terms of alternative perspectives of their past for their consideration or as narrative experiments in which alternative storylines lead to alternate outcomes. Thus, anchoring points may still be utilized without jeopardizing the narrative's theme of autonomy and self-determination. For these clients, exposure procedures may also provide important opportunities for the therapist to move from a role of outsider to audience, and then from audience to co-author in the narrative process.

Second, for many individuals experiencing posttraumatic symptoms, their associated trauma has as much or more to do with their personal actions during the traumatic event than with the circumstances of the event itself. Such persons may be traumatized by experiencing the self as "perpetrator" or "failure" rather than "victim." For example, it may be difficult to apply BFT with an infantry soldier struggling with intrusive images of an enemy soldier he killed even though the enemy soldier was injured and could have been captured alive. In such cases, the disruption in the soldier's personal narrative may not center around adopting the role of a victim, but instead is confronted with his role as the victimizer. With such clients, it may be difficult to identify anchoring points that communicate the client's preparedness for the traumatic event, without implying they were somehow bred to traumatize others, further solidifying their fears.

Similarly, applying BFT to military personnel may also pose some challenges in that service men and women are likely to have been provided with explicit training regarding the dangerous and horrific events they would almost certainly face. For the individual who, despite his or her training, still experiences the intense fear, horror, and helplessness associated with a traumatic reaction, this can be conceptualized as a failure despite preparation, rather than survival because of preparation. The therapist applying BFT in such a context may need to augment their approach. For these clients, the presence of anchoring points is already explicit 
"Back to the Future" Therapy: Its Present Relevance, Promise, and Implications

within their narrative. In fact, it was those very anchoring points which were put in place to protect and prepare the client that appear to have failed. Presenting additional new anchors may be less effective if those anchors that have been months or years in the making appear to the client to have failed. An approach that reconceptualizes the meaning of the anchor, rather than identifying new ones, may be beneficial.

Finally, individuals engaged in high-risk occupations such as the military have histories in which anchoring points can be clearly identified. The ease of identifying such anchoring points in civilian populations is likely to vary considerably from client to client. Whereas some individuals exposed to traumatic events may have prior experience that lends itself to being written into the narrative as a preparatory anchor, it seems likely that some other clients will have experienced traumatic events without any discernable preparation. In such cases, it is not only important for the therapist to redefine an element of the client's history as an anchoring point, but the therapist must do so in a way the client can accept as a credible, alternative telling of their history. This is a daunting task. Suggesting, for example, that viewing the 9/11 attacks on television prepared someone to face a traumatic event such as an automobile accident or a sexual assault is likely to strain both the client's credulity and the therapist's credibility. The therapeutic creativity and interpersonal sensitivity necessary in such cases would be substantial.

Given that the majority of individuals undergoing traumatic events eventually recover, non-intrusive, resilience-focused interventions such as BFT appear strongly warranted as means to prevent consolidation of traumatic experiences and to reduce the number of individuals experiencing acute stress reactions who then go on to develop PTSD. In the event that a client's traumatic nucleus already appears to have solidified, the authors state directly that traditional cognitive behavioral approaches should be adopted instead of BFT. However, it should be emphasized that narrative approaches to trauma therapy are still applicable to PTSD, even when the traumatic nucleus is fully formed. Rather than automatically referring to non-constructivist orientations, it may be more consistent with BFT's underlying assumptions to consider other narrative approaches to trauma that involve re-narrating core aspects of identity (such as a consolidated traumatic nucleus). Such approaches have been elaborated by Sewell and colleagues (e.g., Sewell, 1997, 2005; Sewell \& Williams, 2001, 2002) directly in relation to PTSD, as well as by Ecker and Hulley $(1996,2008)$ and others more generally.

\section{INVESTIGATING BFT}

As with any therapy, it is crucial for the therapist to know when BFT as a treatment is indicated, and when other approaches might be more appropriate. Of central importance for BFT is the ability to determine if the consolidation of the traumatic nucleus has already occurred for a client. Prior to such consolidation, BFT remains a viable alternative. But once the traumatic nucleus has been solidified, clients would be less likely to adopt anchoring points provided by the therapist as being legitimate narrative alternatives. Thus, determining the applicability of BFT in any given case requires that "consolidation of the traumatic nucleus" be somehow operationalized.

As the authors suggest, the first evidence for a consolidated traumatic nucleus is the time elapsed since the traumatic event. Although time alone should not exclude the possibility of a 
"Back to the Future" Therapy: Its Present Relevance, Promise, and Implications

still unconsolidated traumatic nucleus, time is not on the side of the BFT therapist. Nonetheless, time cannot be considered a precise operational discriminator between those with and without a consolidated traumatic nucleus.

To some extent, the client's own description of symptoms may be a guide as to whether the traumatic nucleus has consolidated. Palgi and Ben-Ezra (2010) define the traumatic nucleus as the "experience, sensory feeling, and/or memory that comprise the essence of the horror." Therefore, if the client describes a general sense of unease, tension or vague memories of intense emotional states, but lacks more concrete indications of the setting, objects, and individuals involved in the traumatic event, then the narrative may still be in a state of flux and BFT may be effective in preventing the traumatic nucleus from solidifying. Alternatively, if the individual's complaints clearly contain concrete, graphic elements (images, smells, sounds) regarding the event (and these are considered by the client the distressing elements), then it may be inferred that consolidation has already taken place and other approaches aimed at reconstructing the traumatic narrative might be considered.

Making this distinction involves a dilemma for the assessing therapist. Inquiring directly after the content of the client's intrusive posttraumatic symptoms is apt to recall them into the client's active memory, risking further consolidation of the traumatic nucleus. To avoid such an event, the therapist engaged with a survivor may need to approach the client's experience with the "clean," neutralized language referred to by Palgi and Ben-Ezra. Furthermore, in exploring the client's status they may simply wish to ask, "What about (the event) is disturbing for you?" and then allow the client to respond naturally. The client may then be more likely to select the elements of the traumatic events that distress them, while remaining relatively free from suggestions that their story should contain a re-telling of the memory itself.

As a new method for the treatment of ASD, BFT presents an opportunity for further empirical investigation. A broader application of BFT to trauma survivors, compared to exposure techniques and wait-list controls following traumatic events may provide insight into BFT's relative efficacy with regard to established treatments. Clearly, operationalizing nonconsolidated traumatic nuclei would be a pre-requisite to such empirical study. But once operationalized, the narrative discussions between the therapists and individuals receiving BFT could be recorded and analyzed for the "anchoring" elements that Palgi and Ben-Ezra describe. These individuals' narratives could be compared to narratives from control and traditional approaches for content and their relation to posttraumatic symptom levels.

\section{SUMMARY}

BFT provides an important treatment option geared towards preventing traumatic narratives from solidifying and creating the ongoing distress associated with chronic PTSD. Although uncommon in its concentration on acute stress reactions, BFT shares similar concepts with other narrative approaches to posttraumatic stress (Sewell \& Williams, 2002; Ecker \& Hulley, 1996). As such, BFT provides an alternative approach to other cognitive and behavioral models of trauma intervention for early responses to trauma. Although approximately 1 out of 10 trauma survivors develop PTSD, one half to three-fourths of individuals with ASD go on to develop chronic PTSD (Bryant, 2003; Bryant et al., 2008). Clearly, this population represents 
"Back to the Future" Therapy: Its Present Relevance, Promise, and Implications

high risk for future distress and impairment, so BFT presents a valuable opportunity for preventing later impairment and suffering. Research into the operationalization of traumatic nucleus consolidation remains crucial in that it will better allow the targeted application of BFT to those trauma survivors who are most likely to benefit, and it will facilitate needed empirical research.

\section{REFERENCES}

Borja, S. E., Callahan, J. L., \& Long, P. J. (2006). Positive and negative adjustment and social support of sexual assault survivors. Journal of Traumatic Stress, 19, 905-914.

Bryant, R. A. (2003). Early predictors of posttraumatic stress disorder. Biological Psychiatry, 53, 789-795.

Bryant, R.A., Creamer, M., O'Donnell, M.L., Silove, D.,\& McFarlane, A.C. (2008). A multisite study of the capacity of acute stress disorder diagnosis to predict posttraumatic stress disorder. Journal of Clinical Psychiatry 69(6), 923-929.

Ecker, B. \& Hulley, L. (1996). Depth oriented brief therapy: How to be brief when you were trained to be brief, and vice versa. San Francisco: Jossey-Bass.

Ecker, B. \& Hulley, L. (2008). Coherence therapy: Swift change at the core of symptom production. In J. D. Raskin \& S. K. Bridges (Eds.), Studies in Meaning 3. New York: Pace University Press.

Eisen, M., \& Lynn, S. (2001). Dissociation, memory and suggestibility in adults and children. Applied Cognitive Psychology, 15(7), S49-S73.

Kelly, G. A. (1955/1991). The psychology of personal constructs (2 vols.). New York: Norton. Reprinted by London: Routledge.

McAdams, D. P. (2006). The problem of narrative coherence. Journal of Constructivist Psychology, 19, 109-125.

Palgi, Y., \& Ben-Ezra, M. (2010) "Back To The Future”: Narrative treatment for post-traumatic, acute stress disorder in the case of paramedic Mr. G. Pragmatic Case Studies in Psychotherapy, Vol. 6(1), Article 1, 1-26 Available: http://hdl.rutgers.edu/1782.1/pcsp_journal

Sewell, K.W. (1997). Posttraumatic stress: Towards a constructivist model of psychotherapy. In R.A. Neimeyer \& G.J. Neimeyer (Eds), Advances in personal construct psychology: Volume IV (pp. 207-235). Greenwich, CT: JAI Press.

Sewell, K. W. (2005). Constructivist trauma psychotherapy: A framework for healing. In D. Winter \& L. Viney (Eds.), Personal Construct Psychotherapy: Advances in Theory, Practice, and Research (pp. 165-176). London: Whurr.

Sewell, K.W. \& Williams, A.M. (2001). Construing stress: A constructivist therapeutic approach to posttraumatic stress reactions. In R.A. Neimeyer (Ed.), Meaning reconstruction and the experience of loss (pp. 293-310). Washington, DC: American Psychological Association.

Sewell, K. W. \& Williams, A. M. (2002). Broken narratives: Trauma, metaconstructive gaps, and the audience of psychotherapy. Journal of Constructivist Psychology, 15, 205-218.

Ullman, S., \& Fillipis, H. (2001). Predictors of PTSD symptom severity and social reactions in sexual assault victims. Journal of Traumatic Stress, 14, 369-389.

White, M., \& Epston, D. (1990). Narrative means to therapeutic ends. New York: Norton. 